\title{
USE OF UPPER-ARM ANTHROPOMETRY AS MEASURE OF BODY-COMPOSITION AND NUTRITIONAL ASSESSMENT IN CHILDREN AND ADOLESCENTS (6-20 YEARS) OF ASSAM, NORTHEAST INDIA
}

\author{
Singh Jaswant ${ }^{1}$, Mondal Nitish ${ }^{2}$
}

ABSTRACT

BACKGROUND: Upper-arm muscle area (UMA), upper-arm fat area (UFA), arm-fat index (AFI), upper-arm fat estimate (UFE) and upper-arm muscle estimate (UME) was introduced for the assessment of body-composition. This cross-sectional study assessed age-sex specific upper-arm composition and nutritional status among children and adolescents.

METHODS: The present cross-sectional study was conducted among 1545 (770 boys; 775 girls) Sonowal Kacharis of Dibrugarh District, Assam, Northeast-India, using multi-stage stratified random sampling method. The anthropometric measurements of height, weight, triceps and mid-upper-arm circumference were recorded. The upper-arm composition was assessed using standard equations. Nutritional status was assessed using standard classification of upper-arm muscle-area by height (UAMAH) and thinness (low BMI-for-age).

RESULTS: Age and sex-specific muscularity were found significantly greater among boys than girls $(p<0.01)$, while adiposity was significantly greater among girls $(p<0.01)$, particularly when they approached to puberty. The overall prevalence of low and below-average UAMAH was found to be $16.38 \%$ and $22.65 \%$ respectively. The overall prevalence of thinness was $23.69 \%$ (26.36\% boys, $21.03 \%$ girls) (p>0.05).

CONCLUSION: Body-composition and nutritional status of these children and adolescents were found markedly unsatisfactory using upper-arm composition, UAMAH and thinness. The combination of upper-arm composition and conventional anthropometric measures appear to be useful for bodycomposition and nutritional status assessment.

KEY WORDS: Upper-arm Muscle Area, Upper-arm Fat Area, Upper-arm anthropometry, Upper-arm muscle-area by Height, Northeast-India, Malnutrition, Child Health

\section{DOI: http://dx.doi.org/10.4314/ejhs.v24i3.8}

\section{INTRODUCTION}

Body-composition is of interest to nutritionists and physical anthropologists because of the impact of nutritional status, physical activity, disease, environment and genetic factors. Bodycomposition is extremely difficult to assess with fair accuracy, and several techniques have been developed for an accurate estimation and distribution of adiposity (1-4). In numerous epidemiological and clinical situations, the bodycomposition is determined with available methods, such as bioelectrical impedance analysis, dual-Xray absorptiometry and computerized tomography (2-6). A major difficulty in the interpretation of body-composition analysis is different methods may yield different results for the same variable in individual (7). The body-composition assessment

\footnotetext{
${ }^{1}$ Department of Anthropology, Dibru College, Dibrugrah, Assam, India

${ }^{2}$ Department of Anthropology, Assam University; Diphu Campus, Karbi Anglong, Assam, India

Corresponding Author: Dr. Nitish Mondal, E-mail:nitish_slg@ rediffmail.com
} 
based on anthropometric measurements are still an important method of choice in epidemiological and clinical investigations. Generally, the assessment of muscularity and adiposity are done using skinfolds thickness and circumference measurement (2,4,8-11). Body-composition is most commonly assessed using surrogate anthropometric measures like body mass index (BMI), measures excess adiposity in relation to greater body-weight relative to height rather than excess body-fat (12-13), which is unable to differentiate between excess body-weight associated with muscle-mass and/or fat-mass $(1,3,14)$. The relationship between BMI and bodycomposition and between skinfolds and bodycomposition varies across the populations $(1,4,7)$.

The upper-arm composition is usually assessed using upper-arm muscle area (UMA), upper-arm fat area (UFA), arm-fat index (AFI), upper-arm fat area estimate (UFE) and upper-arm muscle area estimate (UME) calculated from midupper-arm circumference (MUAC) and triceps (TSF) skinfold thickness are used to determine the body-composition $(5,9,11,15-16)$. The upper-arm composition has received considerable attention during the last few decades, but not been adopted widely for assessment of body-composition and nutritional status. The upper-arm-muscle area by height (UAMAH) is derived to assess the nutritional status related to reserve body-protein and longitudinal growth patterns (17). However, very few studies have reported on bodycomposition and nutritional status related to upper-arm composition (18-24). The bodycomposition allows a quantitative assessment of muscle-mass and adiposity changes that reflects nutritional intake, losses and expenses over timeperiod $(1,3,14)$. Recently, several investigations have shown the direct association of disease, biochemical changes, clinical diagnosis and nutritional status with upper-arm composition $(6,25,26)$. Furthermore, age-sex and population specific upper-arm anthropometry seems to be an important technique to determine bodycomposition and nutritional status especially in epidemiological, clinical diagnosis and disease prevalence. It is evident that the body-composition variations are generally attributed to geographic, environmental, genetic and socio-economic factors across populations $(1,3,7,14)$. There is paucity in the age-sex specific changes studies in body-composition (18-24) and standard growth reference for school-age children and adolescents related to upper-arm anthropometry $(8,9,17,20)$. Therefore, the objective of this study is to assess the age-sex specific variation in the upper-arm composition and the related usefulness in assessment of body-composition and nutritional status among children and adolescents (aged 6-20 years) of Assam, Northeast-India.

\section{MATERIAL AND METHODS}

The present cross-sectional study was conducted among 1545 (770 boys; 775 girls) children and adolescents aged 6-20 years belonging to the Sonowal Kachari of Dibrugarh District (Latitude $27^{0} 5^{\prime} 38^{\prime \prime} \mathrm{N}$ to $27^{0} 42^{\prime} 30^{\prime \prime} \mathrm{N}$ and Longitude $94^{0} 33^{\prime} 46^{\prime \prime} \mathrm{E}$ to $95^{0} 29^{\prime} 8^{\prime \prime} \mathrm{E}$ ), Assam, NortheastIndia. Ethnically, they belong to Mongoloid tribal population and shows affinity to Bodo Kachari and Dimasa Kachari of Northeast-India (27). The community has an area of $3381 \mathrm{~km}^{2}$ having a total population of $13,27,748(6,80,114$ males; $6,47,634$ females) with a total literacy rate of $76.22 \%$. According to the National Census 2001, the total population of the Sonowal Kachari is $7.10 \%$ of the total scheduled tribe's population of Assam. The data collection was undertaken during the period of July 2006 to June 2008. A total of 20 lowerprimary and 16 higher-secondary schools from 26 villages were covered using multi-stage stratified random sampling method. The minimum number of subjects required for reliable estimate and assessment was calculated utilizing the standard sample size estimation procedures (28). In this method, the anticipated population proportion of $50 \%$, absolute precision of $3 \%$ and confidence interval of $95 \%$ were considered. Hence, the minimum sample size in this study was estimated to be 1014 subjects. The school records were utilized to ascertain their age which was subsequently verified from their birth certificates. A total of 1715 (858 boys; 857 girls) children belonging to a Sonowal Kachari population aged 6-20 years were identified and approached. Of these, 1715 children, a total of 170 ( 88 boys; 82 girls) whose dates of birth were either not available or inappropriate in the school records and/or were not in the age group of 6-20 years were excluded. Parents of the subjects were informed about the study objectives before 
obtaining of the related information. The subjects were free from any previous histories related to medical and surgical episodes, physical deformity and not suffering from any disease at the time of examination.

Data collection: Socioeconomic and demographic data on education, occupation and family income were collected using a structured schedule. The schedules were completed by both schools and household visits to obtain related data. Socioeconomic status (SES) was evaluated using a modified version of the scale of Kuppuswamy (29). This scale allows determination of SES based on a score calculated from education, occupation and monthly income. It was subsequently observed that all selected subjects were belonged to a lower to middle-SES group. The socio-economic and demographic data were not taken into consideration in further statistical analysis after assessing the SES because children belonged to similar group. The necessary approvals and informed consents were collected from the local village and block level authorities, school authorities and subjects and their parents prior to conducting the study. The study approvals and permissions of the protocols were also obtained from the Dibrugarh University, and the study was conducted in accordance with the ethical guidelines for human experiments as laid down in the Helsinki Declaration (30).

Anthropometric Measurement Collection: The anthropometric measurements of height, weight, MUAC and TSF were recorded using standard procedures (11). Heights of the subjects were recorded with the help of an anthropometer with the head of the subject being held in the Frankfurt Horizontal plane to the nearest $0.10 \mathrm{~cm}$. The weight of the children wearing minimum clothing and with bare feet was recorded using a portable digital weighing machine to the nearest $100 \mathrm{~g}$. The MUAC was taken at the point midway between the acromion and the radiale of the upper-arm using a plastic coated non-stretchable measuring tape on the left side to the nearest $0.10 \mathrm{~cm}$. The TSF was measured using a Harpenden skinfold caliper calibrated to exert a constant pressure of $10 \mathrm{gm} / \mathrm{mm}^{2}$ on the left side to the nearest $0.20 \mathrm{~mm}$. The TSF was measured at least three times and the average taken or until the same value was recorded consecutively. The anthropometric measurements were collected by a single observer
(JS). The technical error measurement $\left\{\mathrm{TEM}=\sqrt{ }\left(\sum \mathrm{D}^{2} / 2 \mathrm{~N}\right), \quad \mathrm{D}=\right.$ difference between the measurements, $\mathrm{N}=$ number of individuals measured \} differences were calculated for testing the co-efficient of reliability $[\mathrm{R}=\{1-$ $\left.(\mathrm{TEM})^{2} / \mathrm{SD}^{2}\right\}, \mathrm{SD}=$ standard deviation] of the obtained anthropometric measurements on 50 subjects using standard procedures (31). Very high values of $\mathrm{R}(>0.974)$ were obtained for TEM analysis and these values were found within the suggested cut-off level (TEM >0.95) (31).

Assessment of body-composition: The upper-arm composition was assessed based on anthropometric measurements of MUAC and TSF utilizing standard equations (8-9). The following equations were used:
a) $\mathrm{UMA} \mathrm{cm}{ }^{2}=\{\text { MUAC- }(\mathrm{TSF} \times \pi)\}^{2}$ $(4 \times \pi)$
b) $\mathrm{UFA} \mathrm{cm} \mathrm{cm}^{2}=\left\{\left(\mathrm{MUAC}^{2} /(4 \times \pi)\right\}\right.$-UMA
c) $\mathrm{AFI}=\mathrm{UFA} /\left\{(\mathrm{MUAC})^{2} /(4 \times \pi)\right\} \times 100$

The body-composition was evaluated using newly proposed anthropometric indices of upperarm composition by Rolland-Cachera et al. (15):

d) $\mathrm{UFE}=\mathrm{MUAC} \times(\mathrm{TSF} / 2)$

e) $\mathrm{UME}=\left\{(\mathrm{MUAC})^{2} /(4 \times \pi)\right\}$-UFE

Assessment of Nutritional status: Nutritional status was determined using the Z-score based classification for UAMAH proposed by Frisancho and Tracer (17). The classification is summarized as:

\begin{tabular}{|l|l|l|}
\hline Nutrition status & Category & Z-score \\
\hline Wasted & Category I & $<-1.60$ \\
\hline Below average & Category II & -1.60 to $<-1.00$ \\
\hline Average & Category III & -1.00 to $<+1.00$ \\
\hline Above average & Category IV & +1.00 to $<+1.60$ \\
\hline High Muscle & Category V & $\geq+1.60$ \\
\hline
\end{tabular}

The age-sex specific BMI was calculated for the assessment of nutritional status (32): BMI $\left(\mathrm{kg} / \mathrm{m}^{2}\right)$ $=$ Weight $(\mathrm{kg}) /$ Height $^{2}\left(\mathrm{~m}^{2}\right)$. The prevalence of thinness (low BMI-for-age) was assessed by following the proposed international BMI cut-off points $(32,33)$. The BMI values were used to determine the definite grades of thinness (GradeIII: severe, Grade-II: moderate, Grade-I: mild), these above classifications are similar to the grades of adult chronic energy deficiency (CED) (32). The CED was the chronic undernutrition classified as BMI found below $18.50 \mathrm{~kg} / \mathrm{m}^{2}, 17.00$ $\mathrm{kg} / \mathrm{m}^{2}$ and $16.00 \mathrm{~kg} / \mathrm{m}^{2}$ categorized mild, moderate and severe respectively (32). In this study, a 
subject was found below the age-sex specific thinness grades I, II and III of cut-offs values of the reference are classified as mild, moderate and severely thin respectively $(32,33)$.

Statistical Analysis: The data were analyzed using the Statistical Package for Social Science (version 16.0). One way analysis of variance (ANOVA) using the Scheffe procedure was done to assess age-and sex-specific differences. Twoway ANOVA was used to control the influence of age-sexes on body-composition. Chi-square $\left(x^{2}\right)$ analysis was done to assess the sex-differences in the prevalence of nutritional status with respect to the different nutritional measures. A p-value of $<0.05$ was considered to be statistically significant.

\section{RESULTS}

The descriptive statistics of anthropometric and derived body-composition variables of the Sonowal Kachari boys and girls are shown in Table 1 and Table 2 respectively. The boys are found taller and heavier than girls and mean weight, height and MUAC was gradually increases with ages $(p<0.01)$. The age and sexspecific mean TSF did not show any age-specific trends, but the proportions are significantly greater among girls than boys $(\mathrm{p}<0.05)$. The mean differences are markedly greater in the commencement of puberty and onwards among girls (e.g., 11 years). Age-and sex-specific mean BMI values were increasing with age with the exception in 20 years (in boys) and 8 years (in girls). Using ANOVA, the age-specific mean differences were found significant in weight, height, MUAC, TSF and BMI $(\mathrm{p}<0.01)$. Sexdifferences were found statistically significant $(\mathrm{p}<0.01)$ in height $(\mathrm{F}-\mathrm{value}=15.13)$, TSF $(\mathrm{F}-$ value $=771.67)$ and $\mathrm{BMI}(\mathrm{F}$-value $=24.53)$ with the exception ( $p>0.05)$ observed in weight (Fvalue $=1.10$ ) and MUAC (F-value=0.01). The results of the two-way ANOVA showed significant $(\mathrm{p}<0.01)$ increases with respect to ageand sex in height $(\mathrm{F}$-value $=21.10)$, weight $(\mathrm{F}$ value $=8.74)$, MUAC (F-value $=7.06)$, TSF $(\mathrm{F}-$ value $=35.25)$ and $\mathrm{BMI}(\mathrm{F}$-value $=6.96)$.

Assessment of upper-arm composition: Age-and sex-specific mean UMA and UME values were found significantly greater among boys than girls and gradually increases with ages $(\mathrm{p}<0.01)$, with the only exception in 7 years (Table 1 and 2). Age-specific mean values of UFA and UFE were found significantly greater among girls than boys $(\mathrm{p}<0.01)$. Mean values were not showing any agespecific trends but marked difference was observed in adiposity pattern when approached to puberty especially in girls $(\mathrm{p}<0.01)$. Similarly, mean AFI did not show any specific trends but values were found distinctly greater among girls $(\mathrm{p}<0.01)$. Age-specific mean differences between ages in upper-arm composition variables were found statistically significant in both sexes $(\mathrm{p}<0.01)$. Using ANOVA, sex difference in mean values were found statistically significant $(p<0.01)$ in UMA (F-value=130.14), UFA (Fvalue $=450.25)$, AFI $(\mathrm{F}$-value $=1308.56)$, UME $(\mathrm{F}-$ value $=189.93)$ and UFE $(F-$ value $=469.70)$. There appears to be the existence of a sexual dimorphism in UMA, UFA and AFI measurements. The results of the two-way ANOVA showed statistically significant $(p<0.01)$ increases for UMA (Fvalue $=45.95)$, UFA $(\mathrm{F}$-value $=27.60)$, AFI $(\mathrm{F}-$ value $=41.55)$, UME $(\mathrm{F}$-value $=55.53)$ and $\mathrm{UFE}$ $(F-$ value $=27.73)$ with respect to age and sex. 
Table 1: Age- specific subject distribution, descriptive statistics of anthropometric and body-composition variables among Sonowal Kachari boys.

\begin{tabular}{|c|c|c|c|c|c|c|c|c|c|c|c|}
\hline $\begin{array}{c}\text { Age } \\
\text { (years) }\end{array}$ & $\begin{array}{c}\text { Sample } \\
(\mathrm{N}=770)\end{array}$ & $\begin{array}{c}\text { Weight } \\
\text { (kg) }\end{array}$ & $\begin{array}{c}\text { Height } \\
\text { (cm) }\end{array}$ & $\begin{array}{c}\text { MUAC } \\
(\mathbf{c m})\end{array}$ & $\begin{array}{c}\text { TSF } \\
(\mathbf{m m})\end{array}$ & $\underset{\left(\mathrm{kg} / \mathrm{m}^{2}\right)}{\text { BMI }}$ & $\begin{array}{l}\text { UMA } \\
\left(\mathrm{cm}^{2}\right)\end{array}$ & $\begin{array}{l}\text { UFA } \\
\left(\mathbf{c m}^{2}\right)\end{array}$ & AFI & $\begin{array}{l}\text { UME } \\
\left(\mathrm{cm}^{2}\right)\end{array}$ & $\begin{array}{l}\text { UFE } \\
\left(\mathrm{cm}^{2}\right)\end{array}$ \\
\hline 6 & 50 & $\begin{array}{l}16.17 \\
\pm 1.46 \\
\end{array}$ & $\begin{array}{c}106.66 \\
\pm 3.87 \\
\end{array}$ & $\begin{array}{l}15.49 \\
\pm 0.86 \\
\end{array}$ & $\begin{array}{r}7.19 \\
\pm 1.32 \\
\end{array}$ & $\begin{array}{l}14.21 \\
\pm 0.99 \\
\end{array}$ & $\begin{array}{l}13.97 \\
\pm 1.45 \\
\end{array}$ & $\begin{array}{c}5.19 \\
\pm 1.10 \\
\end{array}$ & $\begin{array}{r}26.92 \\
\pm 3.88 \\
\end{array}$ & $\begin{array}{l}13.55 \\
\pm 1.43 \\
\end{array}$ & $\begin{array}{c}5.61 \\
\pm 1.26 \\
\end{array}$ \\
\hline 7 & 55 & $\begin{array}{l}18.71 \\
\pm 1.97\end{array}$ & $\begin{array}{c}114.58 \\
\pm 4.74\end{array}$ & $\begin{array}{l}15.78 \\
\pm 0.78\end{array}$ & $\begin{array}{c}6.62 \\
\pm 1.39\end{array}$ & $\begin{array}{l}14.23 \\
\pm 0.98\end{array}$ & $\begin{array}{l}14.97 \\
\pm 1.67\end{array}$ & $\begin{array}{c}4.88 \\
\pm 1.06\end{array}$ & $\begin{array}{l}24.56 \\
\pm 4.54\end{array}$ & $\begin{array}{l}14.61 \\
\pm 1.71\end{array}$ & $\begin{array}{c}5.24 \\
\pm 1.21\end{array}$ \\
\hline 8 & 51 & $\begin{array}{l}20.67 \\
\pm 2.61\end{array}$ & $\begin{array}{l}119.93 \\
\pm 5.47\end{array}$ & $\begin{array}{l}16.32 \\
\pm 1.20\end{array}$ & $\begin{array}{r}6.59 \\
\pm 1.62\end{array}$ & $\begin{array}{l}14.32 \\
\pm 0.97\end{array}$ & $\begin{array}{l}16.25 \\
\pm 2.39\end{array}$ & $\begin{array}{c}5.07 \\
\pm 1.44\end{array}$ & $\begin{array}{l}23.61 \\
\pm 4.67\end{array}$ & $\begin{array}{l}15.88 \\
\pm 2.37\end{array}$ & $\begin{array}{c}5.43 \\
\pm 1.62\end{array}$ \\
\hline 9 & 50 & $\begin{array}{l}23.90 \\
\pm 3.04\end{array}$ & $\begin{array}{c}126.12 \\
\pm 6.26\end{array}$ & $\begin{array}{l}16.77 \\
\pm 0.89\end{array}$ & $\begin{array}{c}6.11 \\
\pm 1.37\end{array}$ & $\begin{array}{l}14.97 \\
\pm 0.97\end{array}$ & $\begin{array}{l}17.59 \\
\pm 2.11\end{array}$ & $\begin{array}{c}4.83 \\
\pm 1.11\end{array}$ & $\begin{array}{l}21.53 \\
\pm 4.26\end{array}$ & $\begin{array}{l}17.29 \\
\pm 2.15\end{array}$ & $\begin{array}{c}5.14 \\
\pm 1.25 \\
\end{array}$ \\
\hline 10 & 50 & $\begin{array}{l}26.23 \\
\pm 3.32 \\
\end{array}$ & $\begin{array}{c}131.32 \\
\pm 4.78 \\
\end{array}$ & $\begin{array}{l}17.46 \\
\pm 1.25 \\
\end{array}$ & $\begin{array}{c}6.59 \\
\pm 2.30 \\
\end{array}$ & $\begin{array}{l}15.16 \\
\pm 1.17 \\
\end{array}$ & $\begin{array}{l}18.91 \\
\pm 2.47 \\
\end{array}$ & $\begin{array}{c}5.46 \\
\pm 2.23 \\
\end{array}$ & $\begin{array}{l}22.05 \\
\pm 5.58 \\
\end{array}$ & $\begin{array}{l}18.53 \\
\pm 2.47 \\
\end{array}$ & $\begin{array}{c}5.84 \\
\pm 2.61 \\
\end{array}$ \\
\hline 11 & 51 & $\begin{array}{l}29.94 \\
\pm 4.72\end{array}$ & $\begin{array}{l}137.76 \\
\pm 8.17\end{array}$ & $\begin{array}{l}18.40 \\
\pm 1.49\end{array}$ & $\begin{array}{c}7.05 \\
\pm 2.35\end{array}$ & $\begin{array}{l}15.70 \\
\pm 1.35\end{array}$ & $\begin{array}{l}20.96 \\
\pm 3.30\end{array}$ & $\begin{array}{c}6.14 \\
\pm 2.37\end{array}$ & $\begin{array}{l}22.40 \\
\pm 5.36\end{array}$ & $\begin{array}{l}20.53 \\
\pm 3.32\end{array}$ & $\begin{array}{c}6.57 \\
\pm 2.79\end{array}$ \\
\hline 12 & 51 & $\begin{array}{l}33.44 \\
\pm 5.54\end{array}$ & $\begin{array}{c}143.28 \\
\pm 7.39\end{array}$ & $\begin{array}{l}19.05 \\
\pm 1.96\end{array}$ & $\begin{array}{c}7.57 \\
\pm 2.98\end{array}$ & $\begin{array}{l}16.18 \\
\pm 1.39\end{array}$ & $\begin{array}{l}22.27 \\
\pm 4.12\end{array}$ & $\begin{array}{c}6.88 \\
\pm 3.53\end{array}$ & $\begin{array}{l}23.03 \\
\pm 6.15\end{array}$ & $\begin{array}{l}21.77 \\
\pm 3.97\end{array}$ & $\begin{array}{r}7.40 \\
\pm 4.09\end{array}$ \\
\hline 13 & 52 & $\begin{array}{l}38.89 \\
\pm 5.35\end{array}$ & $\begin{array}{c}150.49 \\
\pm 7.13\end{array}$ & $\begin{array}{l}20.70 \\
\pm 1.53\end{array}$ & $\begin{array}{c}7.43 \\
\pm 2.10\end{array}$ & $\begin{array}{l}17.10 \\
\pm 1.23\end{array}$ & $\begin{array}{l}27.00 \\
\pm 4.48\end{array}$ & $\begin{array}{c}7.26 \\
\pm 2.14\end{array}$ & $\begin{array}{l}21.20 \\
\pm 5.20\end{array}$ & $\begin{array}{l}26.53 \\
\pm 4.54 \\
\end{array}$ & $\begin{array}{r}7.73 \\
\pm 2.44\end{array}$ \\
\hline 14 & 51 & $\begin{array}{l}45.83 \\
\pm 7.93 \\
\end{array}$ & $\begin{array}{c}160.20 \\
\pm 8.69 \\
\end{array}$ & $\begin{array}{l}22.54 \\
\pm 2.40 \\
\end{array}$ & $\begin{array}{r}7.47 \\
\pm 2.09 \\
\end{array}$ & $\begin{array}{l}17.74 \\
\pm 1.86 \\
\end{array}$ & $\begin{array}{r}32.83 \\
\pm 7.03 \\
\end{array}$ & $\begin{array}{r}8.06 \\
\pm 2.81 \\
\end{array}$ & $\begin{array}{r}19.68 \\
\pm 4.39 \\
\end{array}$ & $\begin{array}{l}32.35 \\
\pm 6.98 \\
\end{array}$ & $\begin{array}{r}8.53 \\
\pm 3.13 \\
\end{array}$ \\
\hline 15 & 50 & $\begin{array}{l}48.53 \\
\pm 6.76 \\
\end{array}$ & $\begin{array}{c}162.23 \\
\pm 7.37 \\
\end{array}$ & $\begin{array}{l}23.51 \\
\pm 1.97 \\
\end{array}$ & $\begin{array}{c}8.11 \\
\pm 2.70\end{array}$ & $\begin{array}{l}18.37 \\
\pm 1.69\end{array}$ & $\begin{array}{l}35.20 \\
\pm 5.81\end{array}$ & $\begin{array}{c}9.09 \\
\pm 3.44\end{array}$ & $\begin{array}{l}20.31 \\
+5.33 \\
\end{array}$ & $\begin{array}{l}34.62 \\
\pm 5.79 \\
\end{array}$ & $\begin{array}{c}9.66 \\
\pm 3.88 \\
\end{array}$ \\
\hline 16 & 51 & $\begin{array}{l}50.35 \\
\pm 5.14\end{array}$ & $\begin{array}{c}162.94 \\
\pm 5.38\end{array}$ & $\begin{array}{l}23.82 \\
\pm 2.09\end{array}$ & $\begin{array}{c}8.14 \\
\pm 2.85\end{array}$ & $\begin{array}{l}18.95 \\
\pm 1.50\end{array}$ & $\begin{array}{l}36.22 \\
\pm 6.07\end{array}$ & $\begin{array}{c}9.26 \\
\pm 3.73\end{array}$ & $\begin{array}{l}20.07 \\
\pm 5.59\end{array}$ & $\begin{array}{l}35.64 \\
\pm 6.03\end{array}$ & $\begin{array}{c}9.85 \\
\pm 4.19\end{array}$ \\
\hline 17 & 52 & $\begin{array}{l}51.17 \\
\pm 3.62\end{array}$ & $\begin{array}{c}163.41 \\
\pm 3.81\end{array}$ & $\begin{array}{l}24.09 \\
\pm 1.76\end{array}$ & $\begin{array}{c}6.72 \\
\pm 1.24\end{array}$ & $\begin{array}{l}19.16 \\
\pm 1.20\end{array}$ & $\begin{array}{l}38.64 \\
\pm 5.80\end{array}$ & $\begin{array}{c}7.77 \\
\pm 1.64\end{array}$ & $\begin{array}{l}16.77 \\
\pm 2.70\end{array}$ & $\begin{array}{l}38.28 \\
\pm 5.78\end{array}$ & $\begin{array}{c}8.14 \\
\pm 1.78\end{array}$ \\
\hline 18 & 56 & $\begin{array}{l}53.13 \\
\pm 4.81\end{array}$ & $\begin{array}{c}164.06 \\
\pm 4.96\end{array}$ & $\begin{array}{l}24.99 \\
\pm 1.52\end{array}$ & $\begin{array}{c}7.43 \\
\pm 1.93\end{array}$ & $\begin{array}{l}19.71 \\
\pm 1.21\end{array}$ & $\begin{array}{l}40.98 \\
\pm 4.97\end{array}$ & $\begin{array}{c}8.88 \\
\pm 2.50\end{array}$ & $\begin{array}{l}17.72 \\
\pm 3.90\end{array}$ & $\begin{array}{l}40.52 \\
\pm 4.98\end{array}$ & $\begin{array}{c}9.35 \\
\pm 2.76\end{array}$ \\
\hline 19 & 50 & $\begin{array}{l}54.32 \\
\pm 3.80 \\
\end{array}$ & $\begin{array}{c}164.93 \\
\pm 3.17 \\
\end{array}$ & $\begin{array}{l}25.55 \\
\pm 1.46 \\
\end{array}$ & $\begin{array}{c}7.14 \\
\pm 1.84 \\
\end{array}$ & $\begin{array}{l}19.96 \\
\pm 1.16 \\
\end{array}$ & $\begin{array}{r}43.38 \\
\pm 5.28 \\
\end{array}$ & $\begin{array}{c}8.72 \\
\pm 2.31 \\
\end{array}$ & $\begin{array}{r}16.73 \\
+3.90 \\
\end{array}$ & $\begin{array}{l}42.95 \\
\pm 5.32 \\
\end{array}$ & $\begin{array}{c}9.15 \\
\pm 2.54 \\
\end{array}$ \\
\hline 20 & 50 & $\begin{array}{l}54.98 \\
\pm 5.10\end{array}$ & $\begin{array}{c}165.96 \\
\pm 5.42\end{array}$ & $\begin{array}{l}25.81 \\
\pm 1.17\end{array}$ & $\begin{array}{c}7.39 \\
\pm 1.46\end{array}$ & $\begin{array}{l}19.94 \\
\pm 1.37\end{array}$ & $\begin{array}{l}44.02 \\
\pm 4.75\end{array}$ & $\begin{array}{c}9.08 \\
\pm 1.73\end{array}$ & $\begin{array}{l}17.18 \\
\pm 3.36\end{array}$ & $\begin{array}{r}43.58 \\
\pm 4.82 \\
\end{array}$ & $\begin{array}{c}9.53 \\
\pm 1.91\end{array}$ \\
\hline \multicolumn{2}{|c|}{$\begin{array}{c}\text { F-value } \\
(* p<0.001)\end{array}$} & 483.56 & 619.71 & 309.43 & 3.97 & 149.84 & 313.67 & 27.07 & 21.26 & $312 . .11$ & 22.58 \\
\hline
\end{tabular}

Assessment of Nutritional status: The nutritional status was determined using proposed $\mathrm{z}$-score based UAMAH classification is depicted in Table 3. The results indicated that boys were found more sufferer in undernutrition than girls using UAMAH $(p>0.05)$. The overall prevalence of wasting $(<-1.60 \mathrm{z}$-score $)$ and below average $(-1.60$ to $<-1.00 \mathrm{z}$-score) were found $16.38 \%$ and $22.65 \%$ respectively. The sex-specific prevalence was found to be greater among boys than girls in 'Wasting' (17.32\% vs. 14.84\%) and 'Below average' $(22.38 \%$ vs. $22.33 \%) \quad(\mathrm{p}>0.05)$. The subjects show very less prevalence of high muscle mass of $1.81 \%$ ( $1.68 \%$ boys, $1.94 \%$ girls). Using $\chi^{2}$-analysis, the sex-difference was found insignificant ( $p>0.05)$, except in 'Above average' $(\mathrm{p}<0.05)$. A high prevalence in overall thinness was observed among boys (26.36\%) and girls $(21.03 \%)$. The overall prevalence of mildthinness $(21.43 \%$ vs. $16.13 \%)$ and severe-thinness $(0.77 \%$ vs. $0.65 \%)$ were found greater among boys than girls, with the exception in the moderatethinness $(4.16 \%$ vs. $4.25 \%)$. Sex-difference was found statistically insignificant $(p>0.05)$ with an exception in mild-thinness $(\mathrm{p}<0.05)$ (Table 3). 
Vol. 24, No. 3

July 2014

Table 2: Age- specific subject distribution, descriptive statistics of anthropometric and body-composition variables among Sonowal Kachari girls.

\begin{tabular}{|c|c|c|c|c|c|c|c|c|c|c|c|}
\hline $\begin{array}{c}\text { Age } \\
\text { (years) }\end{array}$ & $\begin{array}{l}\text { Sample } \\
(N=775)\end{array}$ & $\begin{array}{c}\text { Weight } \\
\text { (kg) }\end{array}$ & $\begin{array}{c}\text { Height } \\
(\mathbf{c m})\end{array}$ & $\begin{array}{c}\text { MUAC } \\
(\mathbf{c m})\end{array}$ & $\begin{array}{c}\text { TSF } \\
(\mathbf{m m})\end{array}$ & $\underset{\left(\mathrm{kg} / \mathrm{m}^{2}\right)}{\mathrm{BMI}}$ & $\begin{array}{l}\text { UMA } \\
\left(\mathbf{c m}^{2}\right)\end{array}$ & $\begin{array}{l}\text { UFA } \\
\left(\mathbf{c m}^{2}\right)\end{array}$ & AFI & $\begin{array}{l}\text { UME } \\
\left(\mathrm{cm}^{2}\right)\end{array}$ & $\begin{array}{l}\text { UFE } \\
\left(\mathbf{c m}^{2}\right)\end{array}$ \\
\hline 6 & 50 & $\begin{array}{l}15.51 \\
\pm 1.70\end{array}$ & $\begin{array}{c}105.96 \\
\pm 5.01\end{array}$ & $\begin{array}{l}15.28 \\
\pm 0.92\end{array}$ & $\begin{array}{c}7.80 \\
\pm 1.69\end{array}$ & $\begin{array}{l}13.79 \\
\pm 0.96\end{array}$ & $\begin{array}{l}13.14 \\
\pm 1.54\end{array}$ & $\begin{array}{c}5.51 \\
\pm 1.33\end{array}$ & $\begin{array}{l}29.33 \\
\pm 5.02\end{array}$ & $\begin{array}{l}12.64 \\
\pm 1.56\end{array}$ & $\begin{array}{l}6.01 \\
\pm 1.54\end{array}$ \\
\hline 7 & 51 & $\begin{array}{r}18.73 \\
\pm 2.08\end{array}$ & $\begin{array}{c}113.78 \\
\pm 4.48\end{array}$ & $\begin{array}{l}16.31 \\
\pm 1.20\end{array}$ & $\begin{array}{c}8.07 \\
\pm 2.05\end{array}$ & $\begin{array}{l}14.44 \\
\pm 1.08\end{array}$ & $\begin{array}{l}15.16 \\
\pm 2.00\end{array}$ & $\begin{array}{c}6.12 \\
\pm 1.85\end{array}$ & $\begin{array}{r}28.42 \\
\pm 5.22\end{array}$ & $\begin{array}{l}14.61 \\
\pm 1.97\end{array}$ & $\begin{array}{l}6.67 \\
\pm 2.15\end{array}$ \\
\hline 8 & 53 & $\begin{array}{l}20.19 \\
\pm 2.65\end{array}$ & $\begin{array}{c}119.86 \\
\pm 5.88\end{array}$ & $\begin{array}{l}16.06 \\
\pm 1.14\end{array}$ & $\begin{array}{c}7.19 \\
\pm 1.79\end{array}$ & $\begin{array}{l}14.01 \\
\pm 0.97\end{array}$ & $\begin{array}{l}15.22 \\
\pm 1.85\end{array}$ & $\begin{array}{c}5.42 \\
\pm 1.59\end{array}$ & $\begin{array}{l}25.90 \\
\pm 4.78\end{array}$ & $\begin{array}{l}14.79 \\
\pm 1.80\end{array}$ & $\begin{array}{l}5.85 \\
\pm 1.80\end{array}$ \\
\hline 9 & 50 & $\begin{array}{r}23.73 \\
\pm 3.68\end{array}$ & $\begin{array}{c}126.03 \\
\pm 6.63\end{array}$ & $\begin{array}{l}17.29 \\
\pm 1.38\end{array}$ & $\begin{array}{c}8.24 \\
\pm 2.49\end{array}$ & $\begin{array}{l}14.85 \\
\pm 1.08\end{array}$ & $\begin{array}{l}17.29 \\
\pm 2.55\end{array}$ & $\begin{array}{c}6.65 \\
\pm 2.36\end{array}$ & $\begin{array}{l}27.36 \\
\pm 6.05\end{array}$ & $\begin{array}{l}16.71 \\
\pm 2.54\end{array}$ & $\begin{array}{l}7.23 \\
\pm 2.75\end{array}$ \\
\hline 10 & 53 & $\begin{array}{r}26.25 \\
+3.14 \\
\end{array}$ & $\begin{array}{r}132.67 \\
\pm 5.28 \\
\end{array}$ & $\begin{array}{l}17.67 \\
\pm 1.07 \\
\end{array}$ & $\begin{array}{r}8.90 \\
\pm 2.18 \\
\end{array}$ & $\begin{array}{r}14.88 \\
\pm 1.19 \\
\end{array}$ & $\begin{array}{r}17.66 \\
\pm 1.98 \\
\end{array}$ & $\begin{array}{r}7.28 \\
\pm 1.99 \\
\end{array}$ & $\begin{array}{l}28.90 \\
+5.47 \\
\end{array}$ & $\begin{array}{l}17.00 \\
\pm 2.01 \\
\end{array}$ & $\begin{array}{l}7.94 \\
\pm 2.32 \\
\end{array}$ \\
\hline 11 & 52 & $\begin{array}{l}31.74 \\
\pm 5.40\end{array}$ & $\begin{array}{c}139.83 \\
\pm 6.86\end{array}$ & $\begin{array}{l}19.18 \\
\pm 1.79\end{array}$ & $\begin{array}{l}10.03 \\
\pm 3.84\end{array}$ & $\begin{array}{l}16.15 \\
\pm 1.86\end{array}$ & $\begin{array}{l}20.51 \\
\pm 2.51\end{array}$ & $\begin{array}{c}9.01 \\
\pm 4.35\end{array}$ & $\begin{array}{l}29.52 \\
\pm 6.72\end{array}$ & $\begin{array}{l}19.60 \\
\pm 2.45\end{array}$ & $\begin{array}{l}9.91 \\
\pm 5.34\end{array}$ \\
\hline 12 & 54 & $\begin{array}{l}38.29 \\
\pm 5.77\end{array}$ & $\begin{array}{c}146.81 \\
\pm 6.22\end{array}$ & $\begin{array}{l}20.72 \\
\pm 1.96\end{array}$ & $\begin{array}{l}13.21 \\
\pm 4.32\end{array}$ & $\begin{array}{l}17.71 \\
\pm 2.00\end{array}$ & $\begin{array}{l}21.94 \\
\pm 3.08\end{array}$ & $\begin{array}{l}12.51 \\
\pm 4.84\end{array}$ & $\begin{array}{l}35.33 \\
\pm 7.70\end{array}$ & $\begin{array}{l}20.43 \\
\pm 3.08\end{array}$ & $\begin{array}{r}14.03 \\
\pm 5.88\end{array}$ \\
\hline 13 & 53 & & & $\begin{array}{l}21.91 \\
\pm 2.30\end{array}$ & $\begin{array}{l}15.68 \\
\pm 6.02\end{array}$ & $\begin{array}{l}18.93 \\
\pm 2.71\end{array}$ & $\begin{array}{l}23.05 \\
\pm 3.08\end{array}$ & $\begin{array}{l}15.56 \\
\pm 7.16\end{array}$ & $\begin{array}{l}38.79 \\
\pm 9.26\end{array}$ & $\begin{array}{l}20.84 \\
\pm 3.54\end{array}$ & $\begin{array}{l}17.77 \\
\pm 9.08\end{array}$ \\
\hline 14 & 50 & & $\begin{array}{c}152.94 \\
\pm 4.56\end{array}$ & $\begin{array}{l}22.34 \\
\pm 1.72\end{array}$ & $\begin{array}{l}16.89 \\
\pm 4.65\end{array}$ & $\begin{array}{l}18.90 \\
\pm 1.69\end{array}$ & $\begin{array}{l}23.21 \\
\pm 3.26\end{array}$ & $\begin{array}{l}16.74 \\
\pm 5.07\end{array}$ & $\begin{array}{l}41.21 \\
\pm 8.17\end{array}$ & $\begin{array}{l}20.81 \\
\pm 3.76\end{array}$ & $\begin{array}{r}19.15 \\
\pm 6.33\end{array}$ \\
\hline 15 & 50 & & & $\begin{array}{l}22.89 \\
\pm 1.72\end{array}$ & $\begin{array}{l}17.25 \\
\pm 4.28\end{array}$ & $\begin{array}{l}19.36 \\
\pm 1.65\end{array}$ & $\begin{array}{l}24.37 \\
\pm 3.02\end{array}$ & $\begin{array}{l}17.55 \\
\pm 5.05\end{array}$ & $\begin{array}{l}41.24 \\
\pm 7.01\end{array}$ & $\begin{array}{l}21.89 \\
\pm 3.26\end{array}$ & $\begin{array}{l}20.03 \\
\pm 6.24\end{array}$ \\
\hline 16 & 55 & $\begin{array}{r}46.88 \\
\pm 3.71\end{array}$ & $\begin{array}{c}153.74 \\
\pm 5.09\end{array}$ & $\begin{array}{l}23.74 \\
\pm 1.72\end{array}$ & $\begin{array}{l}16.50 \\
\pm 4.60\end{array}$ & $\begin{array}{l}19.84 \\
\pm 1.43\end{array}$ & $\begin{array}{l}27.51 \\
\pm 3.58\end{array}$ & $\begin{array}{l}17.55 \\
\pm 5.33\end{array}$ & $\begin{array}{l}38.35 \\
\pm 7.76\end{array}$ & $\begin{array}{l}25.21 \\
\pm 4.09\end{array}$ & $\begin{array}{l}19.86 \\
\pm 6.60\end{array}$ \\
\hline 17 & 52 & $\begin{array}{l}48.34 \\
\pm 4.45\end{array}$ & $\begin{array}{c}154.39 \\
\pm 3.83\end{array}$ & $\begin{array}{l}23.94 \\
\pm 1.58\end{array}$ & $\begin{array}{l}15.52 \\
\pm 3.30\end{array}$ & $\begin{array}{l}20.27 \\
\pm 1.64\end{array}$ & $\begin{array}{l}28.99 \\
\pm 2.98\end{array}$ & $\begin{array}{l}16.81 \\
\pm 4.21\end{array}$ & $\begin{array}{r}36.25 \\
\pm 5.45\end{array}$ & $\begin{array}{l}27.02 \\
\pm 2.91\end{array}$ & $\begin{array}{c}18.78 \\
5.01\end{array}$ \\
\hline 18 & 50 & $\begin{array}{r}49.82 \\
\pm 3.95\end{array}$ & $\begin{array}{c}155.12 \\
\pm 4.61\end{array}$ & $\begin{array}{l}24.14 \\
\pm 1.35\end{array}$ & $\begin{array}{l}15.61 \\
\pm 3.05\end{array}$ & $\begin{array}{l}20.69 \\
\pm 1.04\end{array}$ & $\begin{array}{l}29.51 \\
\pm 3.12\end{array}$ & $\begin{array}{r}16.99 \\
\pm 3.75\end{array}$ & $\begin{array}{r}36.28 \\
\pm 5.22\end{array}$ & $\begin{array}{l}27.53 \\
\pm 3.25\end{array}$ & $\begin{array}{l}18.98 \\
\pm 4.52\end{array}$ \\
\hline 19 & 51 & $\begin{array}{l}51.27 \\
\pm 2.57\end{array}$ & $\begin{array}{c}155.58 \\
\pm 3.74\end{array}$ & $\begin{array}{l}24.45 \\
\pm 1.56\end{array}$ & $\begin{array}{l}14.91 \\
\pm 2.81\end{array}$ & $\begin{array}{l}21.19 \\
\pm 0.95\end{array}$ & $\begin{array}{l}31.24 \\
\pm 4.51\end{array}$ & $\begin{array}{l}16.51 \\
\pm 3.46\end{array}$ & $\begin{array}{l}34.54 \\
\pm 5.26\end{array}$ & $\begin{array}{l}29.44 \\
\pm 4.67\end{array}$ & $\begin{array}{l}18.32 \\
\pm 4.12\end{array}$ \\
\hline 20 & 51 & $\begin{array}{l}52.48 \\
\pm 4.63\end{array}$ & $\begin{array}{c}156.55 \\
\pm 4.29\end{array}$ & $\begin{array}{l}24.65 \\
\pm 1.30\end{array}$ & $\begin{array}{l}14.15 \\
\pm 2.67\end{array}$ & $\begin{array}{l}21.39 \\
\pm 1.37\end{array}$ & $\begin{array}{l}32.61 \\
\pm 4.20\end{array}$ & $\begin{array}{l}15.87 \\
\pm 3.11\end{array}$ & $\begin{array}{r}32.71 \\
\pm 5.32\end{array}$ & $\begin{array}{l}30.99 \\
\pm 4.44\end{array}$ & $\begin{array}{r}17.50 \\
\pm 3.68\end{array}$ \\
\hline \multicolumn{2}{|c|}{$\begin{array}{c}\text { F-value } \\
(* p<0.001)\end{array}$} & 514.41 & 568.04 & 246.56 & 59.14 & 172.79 & 223.46 & 80.25 & 32.37 & 165.43 & 70.38 \\
\hline
\end{tabular}

Table 3: Assessment of nutritional status using UAMAH and of thinness among Sonowal Kachari children and adolescent boys and girls

\begin{tabular}{|c|c|c|c|c|c|c|c|c|c|}
\hline \multirow{2}{*}{ Sex } & \multicolumn{4}{|c|}{ UAMAH } & \multicolumn{4}{c|}{ Thinness } \\
\cline { 2 - 9 } & $\begin{array}{c}\text { Categor } \\
\text { y-I } \\
\text { (Wasted) }\end{array}$ & $\begin{array}{c}\text { Category- } \\
\text { II } \\
\text { (Below } \\
\text { average) }\end{array}$ & $\begin{array}{c}\text { Category- } \\
\text { III } \\
\text { (Average) }\end{array}$ & $\begin{array}{c}\text { Category- } \\
\text { IV } \\
\text { (Above } \\
\text { average) }\end{array}$ & $\begin{array}{c}\text { Category- } \\
\text { V } \\
\text { (High } \\
\text { muscle) }\end{array}$ & $\begin{array}{c}\text { Severe } \\
\text { (Grade } \\
\text { III) }\end{array}$ & $\begin{array}{c}\text { Moderate } \\
\text { (Grade } \\
\text { II) }\end{array}$ & $\begin{array}{c}\text { Mild } \\
\text { (Grade I) }\end{array}$ & $\begin{array}{c}\text { Overall } \\
\text { Thinness }\end{array}$ \\
\hline Boys & 138 & 177 & 420 & 22 & 13 & 6 & 32 & 165 & 203 \\
$(\mathrm{~N}=770)$ & $(17.32)$ & $(22.38)$ & $(54.55)$ & $(2.86)$ & $(1.68)$ & $(0.77)$ & $(4.16)$ & $(21.43)$ & $(26.36)$ \\
\hline Girls & 115 & 173 & 465 & 7 & 15 & 5 & 33 & 125 & 163 \\
$(\mathrm{~N}=775)$ & $(14.84)$ & $(22.33)$ & $(60.00)$ & $(0.90)$ & $(1.94)$ & $(0.65)$ & $(4.25)$ & $(16.13)$ & $(21.03)$ \\
\hline Total & 253 & 350 & 885 & 29 & 28 & 11 & 65 & 290 & 366 \\
$(\mathrm{~N}=1545)$ & $(16.38)$ & $(22.65)$ & $(57.28)$ & $(1.88)$ & $(1.81)$ & $(0.71)$ & $(4.21)$ & $(18.77)$ & $(23.69)$ \\
\hline$\chi^{2}$-value & $1.93^{*}$ & $0.06^{*}$ & $1.28^{*}$ & $7.71 * *$ & $0.13 *$ & $0.10^{*}$ & $0.01 *$ & $4.87 * *$ & $3.75^{*}$ \\
\hline
\end{tabular}

Values in parenthesis indicate percentages, $* p>0.05, * * p<0.05$ 


\section{DISCUSSION}

Body-composition and nutritional status assessment based on anthropometry is still an important technique of preference and proving increasingly important in epidemiological and clinical investigation $(7,10,11)$. The skinfolds have been simply derived to quantify the amount of muscularity and adiposity $(7,10,11,15,18,24,34)$. The results showed that muscularity were found significantly greater among boys than girls $(\mathrm{p}<0.05)$, while similar trends were reported among Indian (21,23-24), Argentinean (18), South Korean (34), Kenyan (35), Zimbabwean (36) and Turkish children (22). This greater muscularity among boys than girls was probably due to sexrelated effects. The mean values of the Sonowal Kachari children and adolescents were found lower than the Kenyan (35), Zimbabwean (36) and Turkish children (22). The results also showed that girls were found to have greater fat-pattern than boys related to UFA, AFI and UFE $(p<0.01)$ and results were in agreement with reported findings from Turkey (19), Zimbabwe (36) and Indian children (23,24). The comparison with the American reference also suggested being lower body-composition in relation to muscularity and adiposity (9). These differences can be initially attributed to the different associated factors such as genotype, diet and eating habits, physical activity, SES and environmental conditions during childhood (1,7,10,37-39). Moreover, this poor body-composition largely reflects the inadequate nutrition during early-childhood and is likely to be a consequence of well-known phenomenon of prolonged breastfeeding combined with inadequate weaning food of low energy-density (40). Furthermore, it is evident that most of the environmental factors are associated to a large extent with body-composition in children and adolescents, the most important of which are nutrition, disease and infections, and the relative interactions between the two $(1,3,38)$. Additionally, birth-weight, catch-up growth, breastfeeding and early adiposity rebound have impacts on body-composition into childhood and puberty $(1,37,38)$.

Sexual dimorphism in body-composition and fat-patterns are primarily attributed to the action of sex steroid hormones, genetic and/or environmental factors which derives the dimorphism due to these similar changes in bodycomposition during childhood and commencement of puberty $(1,2,7,37,41)$. The changes in bodycomposition characteristics among children and adolescents have been observed in the present study, especially when they approached the stage of puberty. Several studies have reported similar changes in upper-arm composition related to sexual attainment during puberty $(5,16,21,24)$. Several studies have already opined that adiposity showed stability between infancy and childhood and sex-differences appear in body-composition prior to the onset of sexual maturation $(1,2,7)$. The continuation of poor upper-arm composition and high prevalence of undernutrition using UAMAH and thinness among these children and adolescents are probably attributed to poor SES, greater childhood undernutrition and infectious disease prevalence in the vulnerable segments in Indian populations (7,36,40,42-44). Furthermore, early life experiences involving adverse environmental condition, intrauterine growth retardation, poor physical growth during early childhood and subsequent catch-up growth can also impact on growth attainment, body-composition, and health related outcomes later in adulthood $(32,38,45$, 46). It is evident that lower growth attainment in nutritionally vulnerable segments in developing countries including these Sonowal Kachari of Northeast-India, is probably because of the more pronounced influence of specific non-genetic factors such as infectious disease and undernutrition $(7,38,39,45)$. Therefore, bodycomposition and nutritional status evaluation and monitoring should be integrated into routine epidemiological and clinical practices for initial assessment and sequential follow-up to evaluate the effectiveness of implemented and/or ongoing intervention programmes.

The upper-arm composition can provide better assessment of muscularity and adiposity over conventional anthropometric measure, but it is still relatively insensitive to short-term alterations in body-composition $(24,47)$. The UAMAH is considered to be an interesting index used to identify risk factors with chronicundernutrition where both muscle-mass and fatmass are depleted, especially in developing countries when age is either not available or inappropriate (18). The comparison with the reference populations showed these children and 
adolescents were found markedly lower than the American (17) and the Egyptian (20) counterparts indicating poor nutrition (Figure 1). A total of $16.38 \%$ and $22.65 \%$ subjects were found nutritionally vulnerable in 'wasting' and 'below average'. Sen et al. (24) also reported very high prevalence of undernutrition among Bengali Muslim children in West Bengal, India using UAMAH. It is believed that the greater muscularity would reflect a greater protein reserve and lowest musculature is related to lowest height in children $(1,8,9,17)$. A significantly higher prevalence of thinness was found using thinness than UAMAH $(\mathrm{p}<0.01)$. Similar prevalence of thinness among Indian children and adolescents has been reported in Andhra Pradesh (20.6\%) (43), North-Indian (30.6\%) (44) and West Bengal (20.2\%) (48), but found distinctly lower than rural adolescents of Assam (50.2\%) (49) and West Bengal $(42.4 \%)$ (50). The poor nutritional status, particularly girls in the higher ages has important implications in terms of reduced physical-work capacity, poor and adverse reproductive outcomes $(32,46,49,50)$.

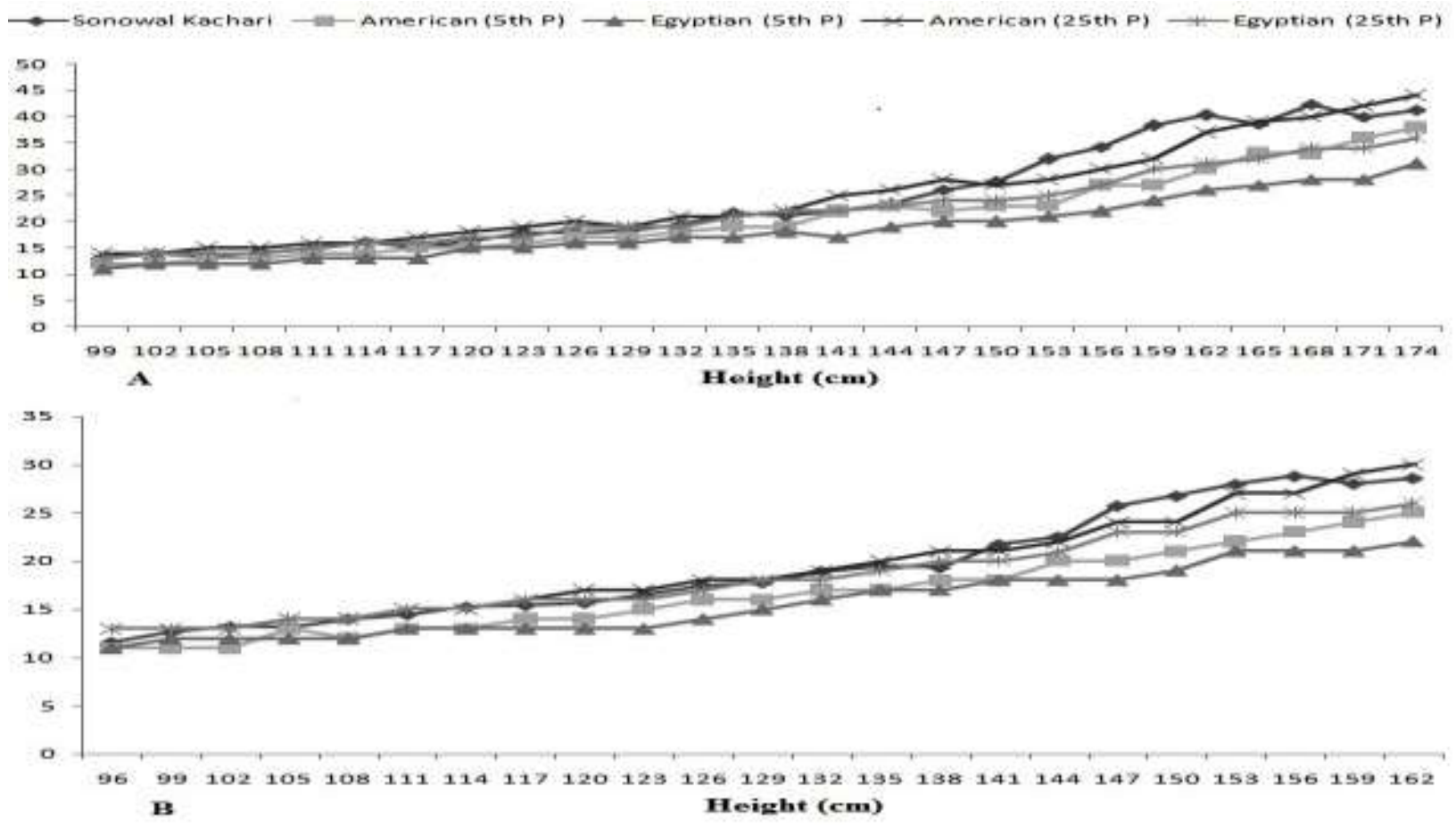

Figure 1: Age and sex specific comparison of mean UAMAH with American and Egyptian children among the Sonowal Kachari Boys (A) and Girls (B)

The combination with upper-arm composition and conventional anthropometric indices appear to be useful for the body-composition and nutritional status assessment $(20,21,24)$. Apparently, the use of UAMAH has improved the accuracy of investigation in undernutrition assessment and hence seems more appropriate indicator of undernutrition. This could allows for an objective, the systematic and early screening of ill-health condition and promote rational and early initiation of optimal support, thereby reducing morbidity, mortality, worsening of the quality of life and global healthcare costs $(3,14,32)$. The changes in muscularity are utilized as a universal index of nutritional status and body-composition where height and UAMAH are more strongly related to muscle-mass rather than to adiposity pattern $(17,20,24)$. Furthermore, it is evident that the body's response to malnutrition followed a hierarchical sequence in which the body-fats and muscles were depleted first and as undernutrition continued, thereby children body-composition was retarded (17). Therefore, the upper-arm anthropometric measures also are very useful to monitor the body-composition, nutritional status and evaluating the effects of intervention and supplementary programmes. Further studies are also needed to confirm the association and clinical 
manifestation with different infectious and communicable diseases with body-composition and nutritional status. The present study recommends to determine the generalizability of the findings of this investigation and the assessment of body-composition and nutritional status to improve screening of undernutrition using upper-arm composition, especially in hospitalized patients and community settings so as to accurately identify the risk of lower or greater adiposity and muscularity, and thereby propose a major opportunity to improve health condition through proper intervention programmes and to reduce the subsequent manifestations.

\section{REFERENCES}

1. Wells JCK. The evolutionary biology of human body fatness: thrift and control. Cambridge: Cambridge University Press, 2010.

2. Taylor RW, Grant AM, Williams SM, Goulding A. Sex differences in regional body fat distribution from pre- to postpuberty. Obesity (Silver Spring) 2010; 18: 1410-1416.

3. Thibault R, Genton L, Pichard C. Body composition: why, when and for who? Clin Nutr 2012; 31: 435-447.

4. Lohman TG, Going SB. Body composition assessment for development of an international growth standard for preadolescent and adolescent children. Food Nutr Bull 2006;27: S314-25.

5. Derman O, Yalcin SS, Kanbur NO, Kinik E. The importance of the measurement of the circumference of arm, arm muscle area and skinfold thickness during puberty. Int J Adolesc Med Health 2002; 14: 193-197.

6. Chomtho S, Fewtrell MS, Jaffe A, Williams JE, Wells JC. Evaluation of arm anthropometry for assessing pediatric body composition: evidence from healthy and sick children. Pediatr Res 2006; 59: 860- 865 .

7. Sen J, Mondal N. Fat mass and fat-free mass as indicators of body composition among Bengalee Muslim children. Ann Hum Biol 2013; 40: 286293.

8. Frisancho AR, New norms of upper limb fat and muscle areas for assessment of nutritional status. Am J Clin Nutr 1981; 34: 2540-2045.

9. Frisancho AR, Anthropometric standard for the assessment of growth and nutrition status. University of Michigan Press: Ann Arbor, MI. 1989.
10. Rolland-Cachera MF. Body composition during adolescence: methods, limitations and determinants. Horm Res 1993; 39: S25-S40.

11. Gibson R S. Principle of nutritional assessment. Oxford: Oxford University Press, 2005.

12. Cole TJ, Faith MS, Pietrobelli A, Heo M. What is the best measure of adiposity change in growing children: BMI, BMI \%, BMI z-score or BMI centile? Eur J Clin Nutr 2005; 59: 419-425.

13. Freedman DS, Wang J, Maynard LM, Thornton JC, Mei Z, Pierson RN, et al. Relation of BMI to fat and fat-free mass among children and adolescents. Int J Obes (Lond) 2005; 29: 1-8.

14. Thibault R, Pichard C. The evaluation of body composition: a useful tool for clinical practice. Ann Nutr Metab 2012; 60: 6-16.

15. Rolland-Cachera MF, Brambilla P, Manzoni P, Akrout M, Sironi S, Del Maschio A, et al. Body composition assessed on the basis of arm circumference and triceps skinfold thickness: a new index validated in children by magnetic resonance imaging. Am J Clin Nutr 1997; 65: 1709-1713.

16. Derman O, Yalcin SS, Kanbur NO, Kinik E. The influence of the sexual stages of adolescent boys on the circumference of the arm, muscle area and skinfold measurements. Int J Adolesc Med Health 2002; 14: 19-26.

17. Frisancho AR, Tracer DP. Standards of arm muscle by stature for the assessment of nutritional status of children. Am J Phys Anthropol 1987; 73: 459-465.

18. Bolzan A, Guimarey L, Frisancho AR. Study of growth in rural school children from Buenos Aires, Argentina using upper arm muscle area by height and other anthropometric dimensions of body composition. Ann Hum Biol 1999; 26: 185.

19. Gültekin T, Özer BK, Katayama K, Akın G. Agerelated patterns of upper arm muscle and fat area in Turkish children and assessment of nutritional status. Int J Anthropol 2006; 21: 231-239.

20. Monir Z, Galal A, Erfan M, Ruby ME. Assessment of growth and nutritional status of Egyptian children and adolescents, using upper arm muscle area by height. Res J Med Med Sci 2008; 3: 60-66.

21. Chowdhury SD, Ghosh T. The upper arm muscle and fat area of Santal children: an evaluation of nutritional status. Acta Paediatr 2009; 98: 103.

22. Ozturk A, Budak N, Cicek B, Mazicioglu MM, Bayram F, Kurtoglu S. Cross-sectional reference values for mid-upper arm circumference, triceps skinfold thickness and arm fat area of Turkish children and adolescents. Int J Food Sci Nutr 2009; 60: 267-281. 
23. Basu D, Sun D, Banerjee I, Singh M, Kalita JG, Rao VR. Cross-sectional reference values of upper arm anthropometry of the Khasi tribal adolescents of Meghalaya, India. Asia Pac J Clin Nutr 2010; 19: 283-288.

24. Sen J, Mondal N, Dey S. Assessment of the nutritional status of children aged 5-12 years using upper arm composition. Ann Hum Biol 2011; 38: 752-759.

25. Hurtado-López EF, Larrosa-Haro A, VásquezGaribay EM, Macías-Rosales R, Troyo-Sanromán $\mathrm{R}$, Bojórquez-Ramos MC. Liver function test results predict nutritional status evaluated by arm anthropometric indicators. $J \quad$ Pediatr Gastroenterol Nutr 2007; 45: 451-457.

26. Akinbami FO, Hamzat TH, Orimadegun AE, Tongo O, Oyeyemi L, Okafor O, et al. Body mass composition: a predictor of admission outcomes among hospitalized Nigerian under 5 children. Asia Pac J Clin Nutr 2010; 19: 295-300.

27. Sengupta S. Kachari Sonowal. In: K. S. Singh ed. People of India: Assam. Vol. XV Part one. Calcutta Seagull Books, 2003.

28. Lwanga SK, Lemeshow S. Sample size determination in health studies: A Practical Manual. Geneva: World Health Organization, 1991.

29. Mishra D, Singh HP. Kuppuswami's socioeconomic status scale - A revision. Indian $J$ Pediatr 2003; 70: 273-274.

30. Touitou Y, Portaluppi F, Smolensky MH, Rensing L. Ethical principles and standards for the conduct of human and animal biological rhythm research. Chronobiol Int 2004; 21: 161-170.

31. Ulijaszek SJ, Kerr DA. Anthropometric measurement error and the assessment of nutritional status. Br J Nutr 1999; 82: 165-177.

32. World Health Organization. Physical status: the use and interpretation of anthropometry. Technical Report Series No. 854. Geneva: World Health Organization, 1995.

33. Cole TJ, Flegal KM, Nicholls D, Jackson AA. Body mass index cut offs to define thinness in children and adolescents: international survey. BMJ 2007;335:194.

34. Kim KB, French KE, Spurgeon JH. Somatic comparisons at four ages of South Korean females and females of other Asian groups. Am J Hum Biol 1999; 11: 735-744.

35. Semproli S, Gualdi-Russo E. Childhood malnutrition and growth in a rural area of Western Kenya. Am J Phys Anthropol 2007; 132: 463-469.

36. Olivieri F, Semproli S, Pettener D, Toselli S. Growth and malnutrition of rural Zimbabwean children (6-17 years of age). Am J Phys Anthropol 2008; 136: 214-222.
37. Wells JC. Sexual dimorphism of body composition. Best Pract Res Clin Endocrinol Metab 2007; 21: 415-430.

38. Ulijaszek SJ. The international growth standard for children and adolescents project: environmental influences on preadolescent and adolescent growth in weight and height. Food Nutr Bull 2006; 27: S279-294.

39. Butte NF, Garza C, de Onis M. Evaluation of the feasibility of international growth standards for school-aged children and adolescents. J Nutr 2007; 137:153-7.

40. Sen B, Bose K, Shaikh S, Mahalanabis D. Prediction equations for body-fat percentage in Indian infants and young children using skinfold thickness and mid-arm circumference. J Health Popul Nutr 2010; 28: 221-229.

41. Haas JD, Campirano F. Interpopulation variation in height among children 7 to 18 years of age. Food Nutr Bull 2006; 27: S212-223.

42. Nandy S, Irving M, Gordon D, Subramanian SV, Smith GD. Poverty, child undernutrition and morbidity: New evidence from India. Bull World Health Org 2005; 83: 210-216.

43. Prashant K, Shaw C. Nutritional status of adolescent girls from an urban slum area in South India. Indian J Pedtr 2009; 76: 501-4.

44. Malhotra A, Passi SJ. Diet quality and nutritional status of rural adolescent girl beneficiaries of ICDS in North India. Asia Pac J Clin Nutr 2007; 16: $58-16$.

45. Henry CJK, Ulijaszek SJ. Long-term consequences of early environment. Growth, development and the lifespan developmental perspective. Cambridge, UK:Cambridge University Press, 1996.

46. Strickland SS. Functional consequences of adult malnutrition in developing countries: a review. $J$ Physiol Anthropol Appl Human Sci 2002; 21: 1-9.

47. Friedl KE, Westphal KA, Marchitelli LJ, Patton JF, Chumlea WC, Guo SS, Evaluation of anthropometric equations to assess bodycomposition changes in young women. Am J Clin Nutr 2001; 73: 268-275.

48. Maiti SD, Chatterjee K, Jana K, Ghosh D, Paul S. Prevalence of stunting and thinness among early adolescent school girls of Paschim Medinipur district, West Bengal. Inter J Biol Med Res 2011; 2: 781-3.

49. Medhi GK, Hazarika NC, Mahanta J. Nutritional status of adolescents among tea garden workers. Indian J Pediatr 2007 ; 74: 343-7.

50. Mondal N, Sen J. Prevalence of stunting and thinness among rural adolescents of Darjeeling district, West Bengal, India. Italian J Pub Health 2010; 7: 54-61. 
